\title{
Aprendizagem Significativa por meio do Uso de TICs: Levantamento das Produções da Área de Ensino de 2016 a 2018
}

\author{
Jainer Diogo Vieira Matos, ProfEPT/IFGoiano, jainerdiogo@ufg.br \\ José Roberto Cruz e Silva, ProfEPT/IFGoiano, jrcs86@gmail.com \\ Altair Fábio Silvério Ribeiro, ProfEPT/IFGoiano, altair@iftm.edu.br \\ Roberta Martins Mendonça Gomes, ProfEPT/IFGoiano, roberta.gomes04@gmail.com \\ Júlio César Ferreira, ProfEPT/IFGoiano, julio.ferreira@ifgoiano.edu.br \\ Fernando Barbosa Matos, ProfEPT/IFGoiano, fernando.matos@ifgoiano.edu.br
}

\section{Resumo}

As tecnologias de informação e comunicação (TICs) estão sendo cada vez mais empregadas no processo de ensino-aprendizagem. Entretanto, o uso destas tecnologias deve corroborar para a aprendizagem significativa e uma mudança de atitude do estudante. $\mathrm{O}$ presente estudo tem como objetivo realizar um levantamento das publicações da área de ensino no período de 2016 a 2018 relacionadas à utilização de TICs com fim de obter uma aprendizagem significativa. Para isso, utilizou-se uma abordagem qualitativa descritiva com revisão sistemática da literatura. As revistas consultadas possuem estrato qualis A1, A2 e B1 na área de ensino e foram selecionadas utilizando o sistema Qualis-Periódicos da plataforma Sucupira. Os resultados demonstram que a utilização das tecnologias digitais para obtenção de uma aprendizagem significativa tem potencial para atingir os mais diversos níveis de educação e áreas do conhecimento. No entanto, os resultados obtidos demonstram a urgente necessidade de mais estudos com foco em Educação Profissional e Tecnológica.

Palavras-chaves: aprendizagem significativa, tecnologias digitais, ensinoaprendizagem, TIC.

\section{Meaningful Learning through the Use of TICs: Survey of the Production of the Teaching Area for the Period 2016 to 2018}

\begin{abstract}
Information and communication technologies (ICTs) are increasingly being employed in the teaching-learning process. However, the use of these technologies should corroborate for meaningful learning and a change of attitude of the student. This study aims to carry out a survey of publications in the area of teaching in the period from 2016 to 2018 related to the use of ICTs in order to obtain meaningful learning. For this, a descriptive qualitative approach was used with a systematic review of the literature. The journals consulted have stratum qualis A1, A2 and B1 in the teaching area and were selected using the system Qualis-Periodicos of the Sucupira platform. The results show that the use of digital technologies to obtain meaningful learning has the potential to reach the most diverse levels of education and areas of knowledge. However, the results obtained demonstrate the urgent need for further studies focusing on Vocation and Technology Education.
\end{abstract}

Keywords: meaningful learning, digital technologies, teaching-learning, ICT.

\section{Introdução}

No contexto da formação educacional brasileira, em especial no âmbito da Educação Profissional e Tecnológica (EPT), muitos são os desafios a serem superados. A busca por uma formação que capacite o aluno nas diversas áreas do conhecimento, não 
somente nas áreas técnicas, mas também lhes dando embasamento para uma análise crítica do avanço social, parece algo inalcançável e exige empenho e dedicação de docentes e discentes, demonstrando que a EPT ocorre em uma dimensão ampliada em relação à educação propedêutica (AGUIAR, 2016).

De acordo com Rosa Júnior et al. (2011), a EPT acumula as funções de formar cidadãos, função principal de todos os tipos de educação, e de formar mão de obra para o mercado, função exclusiva da EPT, o que corrobora com a dimensão ampliada de formação apontada por Aguiar (2016). Toda essa conjuntura tem sido debatida ao longo dos anos por governantes, professores e pesquisadores, tendo como fruto regulamentações, decretos e leis, que buscam ressignificar o ensino médio, associando este nível de formação ao trabalho, a ciência e a cultura, na perspectiva de alcançar um ensino integrado (RAMOS, 2011).

Tanto a atividade educativa como o trabalho são fatores considerados ontológicos, responsáveis pela configuração da sociedade humana atual e pelo seu desenvolvimento ao longo das eras. De acordo com Saviani (2007), a partir da relação trabalho-educação, forma-se um ser que aprende com o trabalho e ensina com o trabalho em uma dimensão unificada. Dessa forma, o pleno desenvolvimento e a formação integral do ser humano devem estar enfatizadas na relação trabalho-educação, nos conceitos, nas técnicas e tecnologias empregadas no processo de produção.

A EPT tem se destacado frente ao cenário atual, buscando aliar a formação profissional com o embasamento teórico propedêutico. A Lei de Diretrizes Básicas $\mathrm{n}^{\circ}$ 9.394/96 (LDB) impõe que o aluno da EPT deve receber uma formação integral, que o prepare para a vida em todos os sentidos. Nessa perspectiva, os conteúdos abordados devem estar ligados à realidade sociopolítica do educando, de modo que sua formação permita o desenvolvimento de visão crítica, da compreensão das contradições da sociedade e da construção de uma prática profissional comprometida, ética e competente (GÓES et al., 2015).

Outro desafio, apontado por Góes et al. (2015), é o crescente desenvolvimento tecnológico, aliado ao surgimento da sociedade da informação. De acordo com Braga et al. (2015), a sociedade tem usado as TICs para auxiliar em diversas atividades, principalmente para comunicação, trabalho, entretenimento e educação. Segundo Kenski (2012), os meios tecnológicos estão inseridos no cotidiano dos alunos e se apresentam como ferramentas atrativas no processo de articulação do conhecimento, entretanto é necessário o embasamento teórico e pedagógico, para que a utilização das TICs não seja ingênua e que possa gerar uma aprendizagem significativa e uma atitude transformadora no estudante.

Tendo em vista que os meios tecnológicos estão cada vez mais inseridos na realidade dos discentes, Góes e Camargo (2012) relatam o potencial que as TICs possuem de trabalhar como um facilitador no processo de ensino-aprendizagem, corroborando com os pressupostos de Masseto (1997). A principal estratégia é aproximar o conteúdo ministrado à realidade dos estudantes, tornando conteúdos abstratos e complexos mais interessantes, facilitando o aprendizado (GÓES e CAMARGO, 2012).

Para Belhot (1997), a utilização das TICs permite um novo caminho de acesso ao conhecimento, tornando o aluno construtor de seu próprio aprendizado e ativo na busca por informações. De acordo com Lima e Furtado (2011), o uso de softwares educativos contribui para a superação de carências e para o desenvolvimento cognitivo. Adicionalmente, o autor afirma que os softwares podem desenvolver no estudante competências ligadas ao mundo do trabalho e ao convívio social, atuando como ferramentas eficazes na EPT. Outros autores como Guerra (2000) e Góes et al. (2013) 
estão alinhados à perspectiva de que as TICs podem ser usadas para mediar e apoiar o processo de ensino-aprendizagem voltado à EPT, desde que sejam utilizadas incorporadas a metodologias de ensino.

Devido à atualidade do tema e a sua importância, a investigação sobre a utilização das TICs e seus efeitos na aprendizagem apresenta extrema relevância para área de ensino. Neste contexto, o objetivo deste artigo foi investigar a percepção de pesquisadores, membros de corpo permanente em programas de pós-graduação no Brasil da área de ensino, quanto à relação do uso das tecnologias digitais e a geração de uma aprendizagem significativa em estudantes de diferentes níveis de formação, desde a educação básica até o ensino superior. Para tanto, foram selecionados artigos publicados em periódicos da área de ensino classificados pela Coordenação de Aperfeiçoamento de Pessoal de Nível Superior (CAPES) no período compreendido entre 2016 e 2018.

\section{Referencial Teórico}

\subsection{Aprendizagem significativa}

Aprendizagem significativa é o termo central utilizado na teoria cognitivista desenvolvida por Ausubel entre 1968 e 1980 (MOREIRA, 2011). David Ausubel, notório psicólogo cognitivista, está entre os teóricos que se voltaram ao estudo dos processos de cognição humana, os quais dão sentido e significado ao mundo. A aprendizagem é um desses processos cognitivos, onde o indivíduo dá significado ao universo ao seu redor enquanto aprende (BESSA, 2008).

De acordo com Moreira (2012), o que caracteriza a aprendizagem significativa é a interação entre conhecimentos prévios e conhecimentos novos, de modo que a interação tenha caráter não-literal, que não seja ao pé da letra, e não-arbitrária, de modo que a interação ocorra com algum conhecimento relevante já existente na estrutura cognitiva. Dessa forma, a aprendizagem significativa pode ser entendida como a ancoragem de informações novas em conceitos anteriormente elaborados dentro da estrutura cognitiva do indivíduo (MOREIRA, 2011).

Os conhecimentos prévios ou proposições relevantes, que são utilizados dentro da cognição humana para ancorar os novos conhecimentos, são denominados de conhecimentos subsunçores, ideias-âncoras ou apenas subsunçores (MOREIRA, 2012). De acordo com Bessa (2008), os subsunçores agem como elementos facilitadores do processo de aprendizagem. Além dos conhecimentos previamente adquiridos, poderiam ser considerados como subsunçores, os diversos artifícios utilizados durante a aula para auxiliar na organização do conteúdo, tais como: explicações introdutórias, materiais, atividades voltadas à construção de uma ideia inicial, ou seja, tudo o que possa servir para facilitar a aprendizagem, inclusive softwares, objetos de aprendizagem e outras TICs na mesma perspectiva. Segundo Moreira (2012), o processo de interação entre novos e prévios conhecimentos é contínuo, o que causa a modificação das ideias-âncoras, tornando a rede de cognição mais robusta e elaborada.

Segundo Klausen (2017), a aprendizagem significativa ocorre em meio a diversos processos de interação com o conhecimento: explorando, elaborando teses, testando-as, construindo analogias e modelos, criando hipóteses, refletindo sobre elas, etc. Ou seja, durante o processo de aprendizagem, o estudante é protagonista e participa ativamente da construção de seu conhecimento, o qual é ampliado durante o fazer e refazer de conceitos e no contato com diversas experiências e tarefas desafiantes. Corroborando com essa proposta, Rogers (2001) conceitua aprendizagem significativa como aquela que provoca uma modificação no indivíduo, seja no comportamento, na orientação, nas suas atitudes 
ou na personalidade, não se limitando a um aumento de conhecimento, mas que penetra profundamente todas as parcelas da existência humana.

Desse modo, emerge a necessidade da realização de estudos investigativos detalhados voltados à área de ensino em meio aos diversos paradigmas apresentados sobre o processo educativo nacional, ao modelo de educação integral idealizado, à utilização das tecnologias nas salas de aulas e à necessidade de desenvolver nos estudantes uma aprendizagem significativa e transformadora. Tais estudos poderiam embasar políticas públicas e principalmente fomentar a modificação dos sistemas tradicionais de ensino empregados ainda hoje, que se apresentam superados frente ao rápido desenvolvimento tecnológico.

\section{Materiais e Método}

O método utilizado neste estudo foi qualitativo descritivo (PADANOV; FREITAS, 2013), consistindo em uma revisão sistemática da literatura que, segundo Galvão e Pereira (2014, p.183), "trata-se de um tipo de investigação focada em questão bem definida, que visa identificar, selecionar, avaliar e sintetizar as evidências relevantes disponíveis". Assim sendo, esta revisão buscou nas produções da área de ensino, no período de 2016 a 2018, a utilização de TICs na perspectiva do desenvolvimento de aprendizagem significativa nos estudantes. Definida a questão de pesquisa, o processo de revisão seguiu as etapas conforme as premissas de Sampaio e Mancini (2007, p. 86):

\footnotetext{
“... [1)] identificar as bases de dados a serem consultadas ; [2] definir palavraschave e estratégias de busca [; 3)] estabelecer critérios para a seleção dos artigos a partir da busca [; 4)] conduzir busca nas bases de dados escolhidas e com base na(s) estratégia(s) definida(s) [; 5)] Comparar as buscas dos examinadores e definir a seleção inicial de artigos [; 6)] Aplicar os critérios na seleção dos artigos e justificar possíveis exclusões [; 7)] Analisar criticamente e avaliar todos os estudos incluídos na revisão [;8)] Preparar um resumo crítico, sintetizando as informações disponibilizadas pelos artigos que foram incluídos na revisão [; 9)] Apresentar uma conclusão, informando a evidência sobre os efeitos da intervenção".
}

A coleta de dados passou por dois momentos, a saber: 1) a seleção de revistas; e 2) a busca dos artigos. Para a escolha das revistas, utilizou-se o sistema Qualis-Periódicos da Plataforma Sucupira. O Qualis-Periódicos é um dos instrumentos utilizados pelo Conselho Técnico-Científico (CTC-ES) para avaliar a produção intelectual na forma de artigos científicos dos programas de pós-graduação do Brasil. Ou seja, lista os veículos utilizados por eles para divulgar suas produções, sendo esses classificados nos seguintes estratos: A1, A2, B1, B2, B3, B4, B5 e C (BARATA, 2016; CAPES, 2012).

Foram selecionados para este estudo os periódicos com classificação A1, A2 e B1 ligados à área de ensino e classificados no quadriênio de 2013 a 2016. Os estratos A1 e A2 foram escolhidos, pois são tidos como de excelência e os estratos B1 por serem de melhor qualidade (BARATA, 2016), representando os mais elevados níveis de produção científica da área. Considerou-se também, para seleção de revistas, aquelas cujos títulos continham as seguintes palavras: "tecnologia", "tecnologias", "ensino", "informação" e "comunicação", visando aproximar o título das revistas com a temática estudada neste trabalho.

Quanto à seleção dos artigos, deve-se considerar que o sistema Qualis-Periódicos não faz a indexação dos artigos científicos publicados (BARATA, 2016). Por isso, para cada uma das revistas selecionadas, foram realizadas buscas específicas por artigos que relacionassem aprendizagem significativa e TICs. As consultas cruzaram o descritor 
"aprendizagem significativa" com os descritores "tecnologias da informação", "tecnologias digitais", "tecnologia educacional", "tecnologias educacionais" e "software".

Após a seleção inicial dos artigos, partiu-se para análise do título e resumo de cada texto, sendo excluídos os artigos que não se enquadraram aos objetivos da pesquisa. Selecionados os artigos, partiu-se para a análise do método e dos resultados apresentados segundo cada artigo. A análise detalhada buscou identificar o tipo de tecnologia empregada na prática educativa, o nível de educação onde a pesquisa foi aplicada, a disciplina ou o conteúdo trabalhado na prática educativa e se a utilização das TICs proporcionou uma aprendizagem significativa nos educandos. Os resultados do percurso metodológico e da análise dos artigos coletados serão apresentados a seguir.

\section{Resultados e Discussão}

Foram selecionadas 25 revistas como base de dados para a busca de artigos, sendo 2 revistas de estrato A1, 9 de estrato A2 e 14 de estrato B1. No entanto, apenas 13 revistas apresentaram resultados na consulta por artigos, sendo 5 revistas de estrato A2 e 8 de estrato B1. A quantidade total de artigos encontrados foi de 41, sendo 20 de estrato A2 e 21 de estrato B1. A Tabela 1 mostra a relação das revistas com a estratificação no QualisPeriódicos e a quantidade de artigos encontrados.

Tabela 1 - Base de dados: revistas, classificação Qualis-Periódicos e quantidade de artigos coletados.

\begin{tabular}{|c|c|c|}
\hline Revista & Qualis & Quant. de Artigos \\
\hline Interface - Comunicação, Saúde, Educação & A1 & 0 \\
\hline Revista Brasileira de Ensino de Física & A1 & 0 \\
\hline Acta Scientiae: Revista de Ensino de Ciências e Matemática & A2 & 8 \\
\hline Areté - Revista Amazônica de Ensino de Ciências & A2 & 1 \\
\hline Caderno Brasileiro de Ensino de Física & A2 & 1 \\
\hline Ensino em Re-Vista & A2 & 0 \\
\hline Ensino, Saúde e Ambiente & A2 & 0 \\
\hline Investigações em Ensino de Ciências & A2 & 0 \\
\hline Rencima - Revista de Ensino de Ciências e Matemática & A2 & 1 \\
\hline Revista Brasileira de Ensino de Ciência e Tecnologia & A2 & 9 \\
\hline Revista Eletrônica Ensino, Saúde e Ambiente & A2 & 0 \\
\hline Tecnologia Educacional & B1 & 9 \\
\hline Revista Tecnologia e Sociedade & B1 & 1 \\
\hline Ensino de Ciências e Tecnologia em Revista & B1 & 1 \\
\hline Tear - Revista de Educação, Ciência e Tecnologia & B1 & 2 \\
\hline Revista Educação \& Tecnologia & B1 & 1 \\
\hline Revista Brasileira de Educação em Ciência da Informação & B1 & 0 \\
\hline $\begin{array}{l}\text { Revista Iberoamericana de Tecnologia en Educación y Educación en } \\
\text { Tecnología }\end{array}$ & B1 & 0 \\
\hline Revista Ciência e Tecnologia & B1 & 0 \\
\hline Revista de La Facultad de Ciencia y Tecnologia & B1 & 0 \\
\hline RECIIS & B1 & 0 \\
\hline Revista Tecnologias na Educação & B1 & 2 \\
\hline Renote & B1 & 4 \\
\hline Revista de Biotecnologia \& Ciência & B1 & 0 \\
\hline Ensino \& Pesquisa & B1 & 1 \\
\hline
\end{tabular}

Fonte: Autoria própria (2018).

Após a primeira etapa de seleção, realizou-se a leitura dos títulos e resumos de cada texto, excluindo-se os artigos que não atendiam o objeto de estudo deste trabalho. 
No total, 22 artigos foram escolhidos para a análise detalhada e completa. Os motivos para exclusão de artigos foram: (i) uso das TICs sem fundamentação teórica; (ii) termos "aprendizagem" e "significativa" apareceram desconectados, de forma que o estudo não abordou o processo de aprendizagem significativa segundo a teoria de Ausubel; (iii) TICs foram utilizadas apenas para fazer análise dos dados da pesquisa; e (iv) trata-se de uma revisão de literatura ou uma pesquisa exploratória. A Tabela 2 apresenta a relação das revistas com a quantidade de artigos selecionados. Pode-se observar que as revistas "Tecnologia Educacional" e "Renote" se destacam na publicação de artigos relacionados à temática deste estudo.

Tabela 2 - Relação de revista e quantidade de artigos selecionados

\begin{tabular}{clcc}
\hline & \multicolumn{1}{c}{ Revista } & Estrato & $\mathbf{N}^{\circ}$ de artigos \\
\hline I & Areté - Revista Amazônica de Ensino de Ciências & A2 & 1 \\
II & Caderno Brasileiro de Ensino de Física & A2 & 1 \\
III & Rencima - Revista de Ensino de Ciências e Matemática & A2 & 1 \\
IV & Revista Brasileira de Ensino de Ciência e Tecnologia & A2 & 3 \\
V & Tecnologia Educacional & B1 & 7 \\
VI & Ensino de Ciências e Tecnologia em Revista & B1 & 1 \\
VII & Tear - Revista de Educação, Ciência e Tecnologia & B1 & 2 \\
VIII & Revista Tecnologias na Educação & B1 & 2 \\
IX & Renote & B1 & 4 \\
\hline
\end{tabular}

Fonte: Autoria própria (2018).

Tabela 3 - Relação de artigos por revista e ano de publicação

\begin{tabular}{|c|c|c|c|}
\hline & Artigo & Ano & Revista \\
\hline 1 & $\begin{array}{l}\mathrm{O} \text { uso de jogos e simulação computacional como instrumento de } \\
\text { aprendizagem: campeonato de aviões de papel e o ensino de Hidrodinâmica. }\end{array}$ & 2017 & II \\
\hline 2 & $\begin{array}{l}\text { Ensino e Aprendizagem de funções trigonométricas por meio do software } \\
\text { geogebra aliado à modelagem matemática. }\end{array}$ & 2016 & III \\
\hline 3 & $\begin{array}{l}\text { Sequências didáticas eletrônicas para auxiliar na aprendizagem significativa } \\
\text { em conteúdos de Patologia Humana. }\end{array}$ & 2016 & IV \\
\hline 4 & $\begin{array}{l}\text { Exploração de simulações como forma de estimular o aprendizado de } \\
\text { conceitos da Cinemática Escalar. }\end{array}$ & 2017 & IV \\
\hline 5 & $\begin{array}{l}\text { Aprendizagem ativa pelo emprego de mapas conceituais: Pesquisa-ação no } \\
\text { ensino de bioestatística em um curso de enfermagem. }\end{array}$ & 2017 & V \\
\hline 6 & $\begin{array}{l}\text { Sequência didática eletrônica com mapas conceituais como complemento de } \\
\text { estudo em patologia humana. }\end{array}$ & 2016 & $\mathrm{~V}$ \\
\hline 7 & $\begin{array}{l}\text { Uso das tecnologias digitais e ferramenta metacognitiva para facilitar a } \\
\text { aprendizagem no ensino superior. }\end{array}$ & 2016 & $\mathrm{~V}$ \\
\hline 8 & $\begin{array}{l}\text { "Jogo da Radiação": Elaboração de um Recurso Computacional Educativo } \\
\text { articulado ao Contexto Ctsa E A Percepção Docente E Discente. }\end{array}$ & 2016 & VI \\
\hline 9 & $\begin{array}{l}\text { Mapas Conceituais e aprendizagem significativa no ensino médio: Análise } \\
\text { da integração entre atividades experimentais e computacionais na } \\
\text { transferência de energia térmica. }\end{array}$ & 2016 & VIII \\
\hline 10 & $\begin{array}{l}\text { Produção de vídeos educativos com o aparelho de telefone celular: uma } \\
\text { proposta para promover a aprendizagem significativa no ensino de ciências } \\
\text { no ensino fundamental. }\end{array}$ & 2017 & VIII \\
\hline 11 & $\begin{array}{l}\text { Interações em blog sobre astronomia: Inovações tecnológicas, motivação, } \\
\text { apropriação de conceitos e linguagem científica. }\end{array}$ & 2016 & VII \\
\hline 12 & Ensino de eletromagnetismo aplicando álgebra visual no curso técnico. & 2016 & IX \\
\hline 13 & $\begin{array}{l}\text { Ensino personalizado: o MOODLE como ferramenta na busca da } \\
\text { Aprendizagem Significativa. }\end{array}$ & 2016 & IX \\
\hline 14 & $\begin{array}{l}\text { Integrando o software scratch ao ensino da geometria: Um experimento } \\
\text { inicial. }\end{array}$ & 2016 & IX \\
\hline
\end{tabular}

Fonte: Autoria própria (2018). 
Após a análise completa dos 22 artigos selecionados na segunda etapa, outros 8 artigos foram excluídos, pelos seguintes motivos: (i) foram identificados estudos preliminares que visavam a construção de softwares e não a aplicação; (ii) a utilização das TICs se apresentou desconectada dos fundamentos da teoria de aprendizagem significativa; e (iii) alguns artigos tinham como foco a discussão da utilização das TICs por docentes em momentos pedagógicos diversos. A Tabela 3 apresentada anteriormente logo após a Tabela 2, traz a relação dos 14 artigos encontrados, por revista e ano, que foram selecionados para análise completa. Observa-se que a maioria das publicações aconteceu no ano de 2016, apresentando queda nos anos posteriores. Percebe-se também que as revistas "Tecnologia Educacional" e "Renote" ainda possuem o maior número de artigos sobre a temática, mesmo findada a segunda etapa de triagem de artigos.

A síntese das informações analisadas nos artigos pode ser observada na Tabela 4, destacando-se: as tecnologias utilizadas, os níveis de educação explorados, os conteúdos e/ou disciplinas abordados e as conclusões sobre a ocorrência de aprendizagem significativa. Tais dados estão apontados de acorda com cada artigo analisado.

Tabela 4 - Resultados: TICs aplicadas e a geração de aprendizagem significativa

\begin{tabular}{|c|c|c|c|c|}
\hline Art & TIC Utilizada & Nível de Educação & $\begin{array}{l}\text { Disciplina/ } \\
\text { Conteúdo }\end{array}$ & $\begin{array}{c}\text { Aprendizagem } \\
\text { significativa }\end{array}$ \\
\hline 1 & $\begin{array}{l}\text { Sequência didática: jogos, atividades } \\
\text { e um simulador. }\end{array}$ & Ensino Médio. & Física. & Ocorrência. \\
\hline 2 & $\begin{array}{l}\text { Sequência didática utilizando slides, } \\
\text { vídeos e o software Geogebra. }\end{array}$ & Ensino Médio. & Matemática. & Ocorrência. \\
\hline 3 & Sequências Didáticas Digitais. & $\begin{array}{l}\text { Graduação em } \\
\text { Educação Física. }\end{array}$ & $\begin{array}{l}\text { Patologia } \\
\text { Humana. }\end{array}$ & Ocorrência. \\
\hline 4 & Software Modellus. & $\begin{array}{l}\text { Técnico em } \\
\text { Informática. }\end{array}$ & Física. & Ocorrência. \\
\hline 5 & Mapas Conceituais Digitais. & $\begin{array}{l}\text { Graduação em } \\
\text { Enfermagem. }\end{array}$ & $\begin{array}{l}\text { Estatística } \\
\text { (Matemática). }\end{array}$ & $\begin{array}{l}\text { Resultado } \\
\text { Inconclusivo. }\end{array}$ \\
\hline 6 & $\begin{array}{l}\text { Conjunto de aplicações web dando } \\
\text { suporte a sequência didática para } \\
\text { elaboração de Mapa Conceitual. }\end{array}$ & $\begin{array}{l}\text { Graduação em } \\
\text { Educação Física. }\end{array}$ & $\begin{array}{l}\text { Patologia } \\
\text { Humana. }\end{array}$ & Ocorrência. \\
\hline 7 & $\begin{array}{l}\text { Conjunto de aplicações web dando } \\
\text { suporte a sequência didática para } \\
\text { elaboração de Mapa Conceitual. }\end{array}$ & $\begin{array}{l}\text { Graduação em } \\
\text { Educação Física. }\end{array}$ & Câncer. & Ocorrência. \\
\hline 8 & Jogo digital. & Ensino Médio. & Química. & $\begin{array}{l}\text { Resultado } \\
\text { inconclusivo. }\end{array}$ \\
\hline $\begin{array}{c}9 \\
10\end{array}$ & $\begin{array}{l}\text { Simulações computacionais. } \\
\text { Produção de vídeos utilizando }\end{array}$ & $\begin{array}{l}\text { Ensino Médio. } \\
\text { Ensino }\end{array}$ & $\begin{array}{l}\text { Física. } \\
\text { Temas }\end{array}$ & $\begin{array}{l}\text { Ocorrência. } \\
\text { Resultado }\end{array}$ \\
\hline 11 & $\begin{array}{c}\text { celulares. } \\
\text { Blog. }\end{array}$ & $\begin{array}{l}\text { Fundamental. } \\
\text { Ensino } \\
\text { Fundamental. }\end{array}$ & $\begin{array}{c}\text { diversos. } \\
\text { Astronomia. }\end{array}$ & $\begin{array}{l}\text { inconclusivo. } \\
\text { Ocorrência. }\end{array}$ \\
\hline 12 & Objeto de Aprendizagem. & $\begin{array}{l}\text { Técnico em } \\
\text { Telecomunicações. }\end{array}$ & $\begin{array}{l}\text { Física } \\
\text { aplicada. }\end{array}$ & Ocorrência. \\
\hline 13 & Objetos de Aprendizagem. & $\begin{array}{c}\text { Ensino } \\
\text { Fundamental. }\end{array}$ & $\begin{array}{l}\text { Língua } \\
\text { Portuguesa. }\end{array}$ & Ocorrência. \\
\hline 14 & Software Scratch. & $\begin{array}{c}\text { Ensino } \\
\text { Fundamental. }\end{array}$ & Matemática. & $\begin{array}{l}\text { Resultado } \\
\text { inconclusivo. }\end{array}$ \\
\hline
\end{tabular}

Fonte: Autoria própria (2018).

Os resultados do levantamento demonstram que diversos tipos de tecnologias digitais podem ser utilizados e/ou combinadas no processo de ensino-aprendizagem, visando a geração de aprendizagem significativa. No entanto, pode-se notar que objetos de aprendizagem, mapas conceituais digitais e tecnologias apoiadas na web têm ganhado 
maior destaque dentre as práticas educativas no período estudado.

Em relação ao nível de educação em que as TICs foram aplicadas, percebe-se que a maioria dos estudos centra na Educação Básica, sendo 4 relacionadas ao ensino fundamental e 4 ao ensino médio. Todavia, também foram identificados 2 artigos voltados à EPT e 4 artigos relacionados à educação de nível superior. Isso indica que as TICs estão sendo exploradas em qualquer nível educacional apoiando e mediando o processo de ensino-aprendizagem, o que corrobora com os estudos de Góes (2013), Guerra (2000) e Lima e Furtado (2011). Entretanto, deve-se analisar previamente quais as tecnologias apropriadas para cada nível e/ou conteúdo e planejar o seu uso embasado em metodologias ativas de ensino.

Além disso, observa-se baixa quantidade de estudos voltados especificamente à EPT, apenas 2 estudos relevantes foram identificados. Observa-se uma lacuna de estudos, principalmente abordando o ensino de disciplinas e/ou conteúdos ligados às áreas de formação profissional, ou seja, áreas técnicas, o que se apresenta como um campo rico de pesquisa a ser cada vez mais explorado. Quanto às disciplinas ou conteúdos abordados, destaca-se o número de trabalhos voltados à disciplina de física, 3 artigos e para o ensino de matemática, 3 artigos. Contudo, percebe-se uma pluralidade de assuntos tratados, podendo-se observar a existência de publicações utilizando TICs no apoio ao ensino de língua portuguesa, conteúdos voltados à saúde humana, química, astronomia e conteúdos diversos.

Por fim, observa-se que as conclusões de grande parte dos artigos analisados, em um total de 10, aponta a ocorrência de aprendizagem significativa por meio das práticas educativas desenvolvidas aliadas ao emprego de diferentes TICs. Este resultado expressivo vai ao encontro dos estudos de Kenski (2012), Góes e Camargo (2012), Masseto (1997), Belhot (1997), Lima e Furtado (2011), Guerra (2000) e Góes et al. (2013). Contudo, em 4 artigos, 3 voltados a educação básica (dois no ensino fundamental e um ensino médio) e 1 voltado ao ensino superior, os resultados apresentados foram inconclusivos, pois não foi relatada a evidência de aprendizagem significativa nos estudantes. Mesmo assim, um resultado relevante é que nenhum dos artigos analisados neste estudo apontou perdas na aprendizagem com a utilização das TICs, o que evidencia a versatilidade e as possibilidades de uso dessas ferramentas apoiando o processo de ensino-aprendizagem em qualquer nível educacional.

Além disso, pôde-se notar nas conclusões dos estudos, outros ganhos relacionados com a utilização das TICs aliadas ao aprendizado. Tais resultados não estão diretamente ligados ao desenvolvimento de aprendizagem significativa, entretanto, estão relacionados principalmente a habilidades desenvolvidas por meio do uso de TICs, tais como: mais interatividade entre os estudantes, atenção e maior interesse durante as aulas e motivação ao aprendizado a partir da prática pedagógica implementada.

\section{Considerações Finais}

Este estudo foi motivado pelo interesse em traçar um panorama atualizado quanto à utilização de ferramentas digitais (softwares, simulações, objetos de aprendizagem, etc.) no espaço escolar, aliadas e apoiando práticas pedagógicas em sala de aula. Com o aumento do acesso a essas tecnologias, este campo de pesquisa se tornou relevante e passou a ser explorado com mais frequência por estudiosos da área de ensino, principalmente tentando identificar as melhorias do processo de ensino-aprendizagem por meio da aplicação e a utilização das TICs.

É importante ressaltar que somente a utilização de um meio tecnológico não possibilita melhorias no processo de ensino-aprendizagem. É necessário o embasamento 
teórico e uma mudança da prática educativa para que a utilização possa corroborar com uma atitude transformadora e com a aprendizagem significativa de conceitos relevantes para o educando. Neste sentido, a revisão sistemática da literatura realizada mostrou que a utilização das TICs, mediadas com a finalidade de obter uma aprendizagem significativa, tem potencial para atingir os mais diversos níveis de educação e áreas do conhecimento.

No entanto, a pouca representatividade de pesquisas voltadas ao ensino profissionalizante e à EPT demonstra a necessidade do desenvolvimento de investigações neste campo de pesquisa. Os autores sugerem como trabalhos futuros, estudos mais amplos buscando a identificação de lacunas de conhecimento quanto a utilização das TICs no ensino em EPT. Estas pesquisas devem abrir caminho para o desenvolvimento de ferramentas digitais específicas, voltadas às diversas áreas do conhecimento, inclusive abarcando as áreas técnicas, favorecendo a formação de profissionais mais bem capacitados e que possuam um olhar crítico, apto a compreender as transformações sociais e a dinâmica dos sistemas produtivos.

\section{Referências}

AGUIAR, R. F. Docência na educação profissional e tecnológica: influência da formação no processo ensino-aprendizagem. 2016. 147 p. Dissertação (Mestrado Profissional em Educação) - Universidade de Brasília, Brasília. 2016.

BARATA, Rita de Cássia Barradas. Dez coisas que você deveria saber sobre o Qualis. RBPG, v. 13, n. 30, p. 13-40, 2016.

BELHOT, R.V. Reflexões e propostas sobre o "ensinar engenharia" para o século XXI. 1997. 113p. Tese (Livre-docência) - Escola de Engenharia de São Carlos, Universidade de São Paulo. 1997.

BESSA, V. H. Teorias da Aprendizagem. Curitiba: IESDE Brasil S.A, 2008. 204 p.

BRASIL, Constituição; BRASIL. Lei no 9.394, de 20 de dezembro de 1996. Estabelece as diretrizes e bases da educação nacional. Diário Oficial da União, v. 134, n. 248, 1996.

COORDENAÇÃO DE APERFEIÇOAMENTO DE PESSOAL DE NÍVEL SUPERIOR. Critérios de classificação Qualis-ensino. Brasília: CAPES, 2012.

GALVÃO, Taís Freire; PEREIRA, Mauricio Gomes. Revisões sistemáticas da literatura: passos para sua elaboração. Epidemiologia e Serviços de Saúde, v. 23, p. 183-184, 2014.

GUERRA, J. H. L.. Utilização do computador no processo de ensino-aprendizagem: uma aplicação em planejamento e controle da produção. 2000. 168p. Tese de Doutorado. Universidade de São Paulo.

GÓES, F. S. N.; CÔRREA, A. K.; CAMARGO, R. A. A.; HARA C. Y. N. Necessidades de aprendizagem de alunos da Educação Profissional de Nível Técnico em Enfermagem. SIED-EnPED, p. 13, 2012. 
GÓES, Fernanda dos Santos Nogueira de et al. Avaliação de um objeto digital de aprendizagem para a educação profissional de nível médio em enfermagem: apoio ao trabalho docente. Anais, 2013.

GÓES, Fernanda dos Santos Nogueira et al. Necessidades de aprendizagem de alunos da Educação Profissional de Nível Técnico em Enfermagem. Revista Brasileira de Enfermagem, v. 68, n. 1, p. 20-25, 2015.

KENSKI, V. M. Educação e tecnologias: o novo ritmo da informação. Campinas: Editora Papirus, 2012. 141p.

KLAUSEN, L. S. Aprendizagem Significativa: Um desafio. In: EDUCERE - Congresso Nacional de Educação, 13, 2017. Curitiba. Anais [...], Curitiba: PUC-PR,2017. p. 64036411.

LIMA, C. B.; FURTADO, A. O. Avaliação de software educativo para educação profissional: uma abordagem cognitivista. In: II ENINED 2011 - II Encontro Nacional de Informática e Educação, 2011, Cascavel.

MOREIRA, M. A. Teorias de Aprendizagem. São Paulo: E.P.U. Ltda. 2. ed. São Paulo, 2011.

O que é afinal Aprendizagem Significativa?[S1, sn], 2012. Disponível em: https://www.if.ufrgs.br/ moreira/oqueeafinal.pdf. Acesso em: 04/06/2018.

PRODANOV, C. C., FREITAS, E. C. Metodologia do Trabalho Científico: Métodos e Técnicas da Pesquisa e do Trabalho Acadêmico. 2. Ed. Novo Hamburgo: Feevale, 2013.

RAMOS, M. N. O currículo para o ensino médio em suas diferentes modalidades: concepções, propostas e problemas. Educação \& Sociedade, v. 32, n. 116, p.771-788, jul./set. 2011.

ROGERS, C. R. Tornar-se pessoa. 5. Ed. São Paulo: Martins Fontes, 2001. 520 p.

ROSA JÚNIOR, C. A.; ROSA, V. C. M.; MATA, L. A. (2018). Anotações sobre o Processo Ensino-Aprendizagem na Educação Profissional e Tecnológica. In:

SIMPÓSIO NACIONAL DE TECNOLOGIA E SOCIEDADE, 4., 2011, Curitiba. Anais [...]. Curitiba: UTFPR, 2011, p. 1-10. Disponível em: http://www.esocite.org.br/eventos/tecsoc2011/cd-anais/arquivos/pdfs/artigos/gt014anotacoessobre.pdf. Acesso em: 15/05/2018.

SAMPAIO, R.F.; MANCINI, M.C. Estudos de revisão sistemática: um guia para síntese criteriosa da evidência científica. Revista brasileira de fisioterapia, v. 11, n. 1, p. 8389, 2007.

SAVIANI, D. Trabalho e Educação: Fundamentos ontológicos e históricos. Revista Brasileira de Educação, São Paulo, v. 12 n. 34, p. 152-165, jan./abr. 2007. 